\title{
High precision control model of large-sized gantry-type linear motor slider
}

\author{
Tetsuya Ojiro ${ }^{1 *}$, Toshiyuki Tachibana ${ }^{2}$, Hideki Honda 3 , So Watanabe ${ }^{4}$, Hiroshi Hamamatsu ${ }^{5}$ \\ and Kazuhiro Tsuruta ${ }^{1}$
}

\begin{abstract}
Large-sized gantry-type linear motor sliders are widely used in industry (e.g. in liquid crystal panel production equipment). An appropriate control model is important for deriving control methods to improve control performance. Although various control models of large-sized gantry-type linear motor sliders have been developed in previous studies, no results are yet reported regarding control models which precisely reproduce characteristics of a large-sized gantry-type linear motor slider. In general, a method to derive a transfer function by using frequency responses can be employed to obtain an accurate control model, however, large sized gantry-type linear motor sliders have unique characteristics, namely "distortion" and "coupling", thus the transfer function derived using only the frequency response cannot reproduce the experimental results with high accuracy. In this paper we report on a method to obtain a highly precise control model of a large sized gantry-type linear motor slider.
\end{abstract}

Keywords: Large-sized gantry-type linear motor slider, Frequency response, Transfer functions modification

\section{Introduction}

In recent years, number of applications of linear motors in industry has increased $[1,2]$. For example, linear motors are used for carrying thin steel plates in the steel industry [3]. However, to carry heavy loads, the linear motor slider is required to have a sufficient power. When aiming at higher power of a single linear motor, special design and individual manufacturing are required to produce a large-sized motor. Special permanent magnets are used to achieve strong magnetic fields, which with other various issues inevitably contribute to costs [4-6]. Therefore, low-cost gantry type linear motor sliders, which enable high output power, are used. Applications include for example liquid crystal panel production devices $[7,8]$ or feed drives of machining centers [9].

To improve control performance of a gantry-type linear motor slider, it is important to develop a precise control

\footnotetext{
*Correspondence: t-ojiro@ip.kyusan-u.ac.jp

1 Department of Mechanical Engineering, Faculty of Science and Engineering, Kyushu Sangyo University, 2-3-1, Matsukadai, Higashi-ku, Fukuoka 813-8503, Japan

Full list of author information is available at the end of the article
}

model. In the previous studies, a control model of largesized gantry-type linear motor slider was developed by using a spring-mass-damper system [4]. However, all frequency characteristics (e.g. yawing vibration and pitching vibration [10], machine stand vibration [11]) were not considered in the control model, thus the accuracy of the control model was not sufficient. For example, the oscillation phenomenon, which occurs in the actual experimental machine when high feedback gain is applied, is not reproduced with the developed control model. Ref. [10] uses a model that takes pitching and yawing vibrations into consideration. However, in Ref. [10] feedback gain is gradually increased with a goal to the problem each time separately. Therefore, numerous experiments are required to increase feedback gain, which takes time and costs. As a method of deriving a model to be capable of reproducing all frequency responses in a single experiment, a method to derive a transfer function using frequency responses is available. However, large sized gantry-type linear motor sliders have unique characteristics, namely "distortion" and "coupling". Ref. [12] proposes a control model that can reproduce all frequency
Springer Open

(c) The Author(s) 2020. This article is licensed under a Creative Commons Attribution 4.0 International License, which permits use, sharing, adaptation, distribution and reproduction in any medium or format, as long as you give appropriate credit to the original author(s) and the source, provide a link to the Creative Commons licence, and indicate if changes were made. The images or other third party material in this article are included in the article's Creative Commons licence, unless indicated otherwise in a credit line to the material. If material is not included in the article's Creative Commons licence and your intended use is not permitted by statutory regulation or exceeds the permitted use, you will need to obtain permission directly from the copyright holder. To view a copy of this licence, visit http://creativecommons.org/licenses/by/4.0/. 


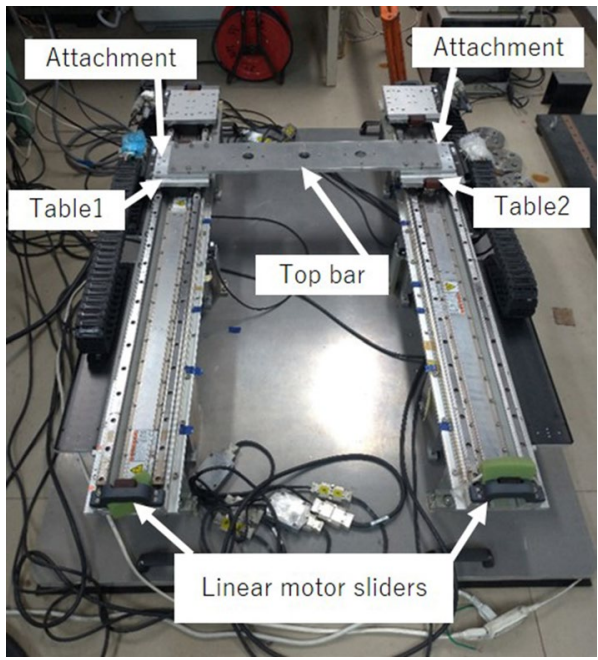

Fig. 1 Gantry-type linear motor slider

Table 1 Specifications of experimental equipment

\begin{tabular}{ll}
\hline Linear slider & SGT1F32-107AH20-0 (YASKAWA) \\
Linear motor & SGLFW-35A120A (YASKAWA) \\
Rated force & $80 \mathrm{~N}$ \\
Max force & $220 \mathrm{~N}$ \\
Table mass & $3.9 \mathrm{~kg}$ \\
Base mass & $42 \mathrm{~kg}$ \\
Stroke & $1070 \mathrm{~mm}$ \\
Distance between two sliders & $500 \mathrm{~mm}$ \\
\hline
\end{tabular}

characteristics and unique characteristics of the large sized gantry-type linear motor slider. However, since Ref. [12] does not consider friction, the experimental results cannot be accurately reproduced. In this paper we demonstrate that the experimental results could be accurately reproduced by considering friction.

\section{Methods/experimental}

\section{Experimental equipment [12]}

Figure 1 shows a large-sized gantry-type linear motor slider which is used in this research. The experimental equipment is comprised of two sliders arranged in parallel and linked by two attachments and a top bar. This experimental equipment has "distortion" and "coupling" which are the problems of large machines [12]. The sliders have linear encoders that measure the position of each table with a resolution of $0.5 \mu \mathrm{m}$. Table 1 shows the main specifications of each linear motor slider.

\section{Control model derivation [12]}

Figure 2 shows a block diagram of the defined control model. Here $f_{1}$ and $f_{2}$ are force references to each linear

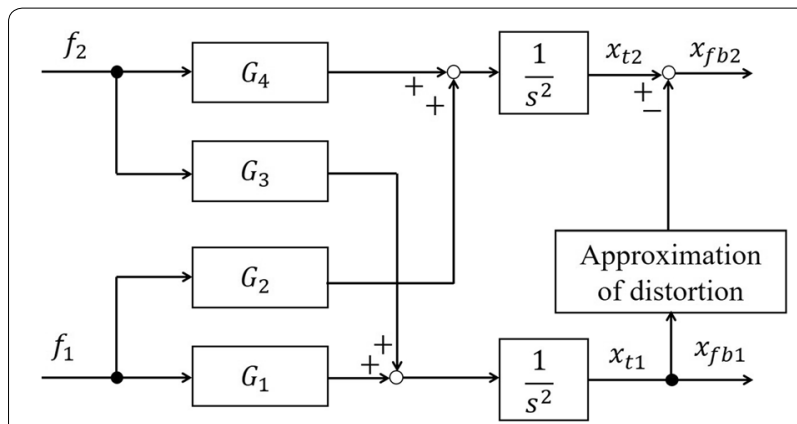

Fig. 2 Identified control model

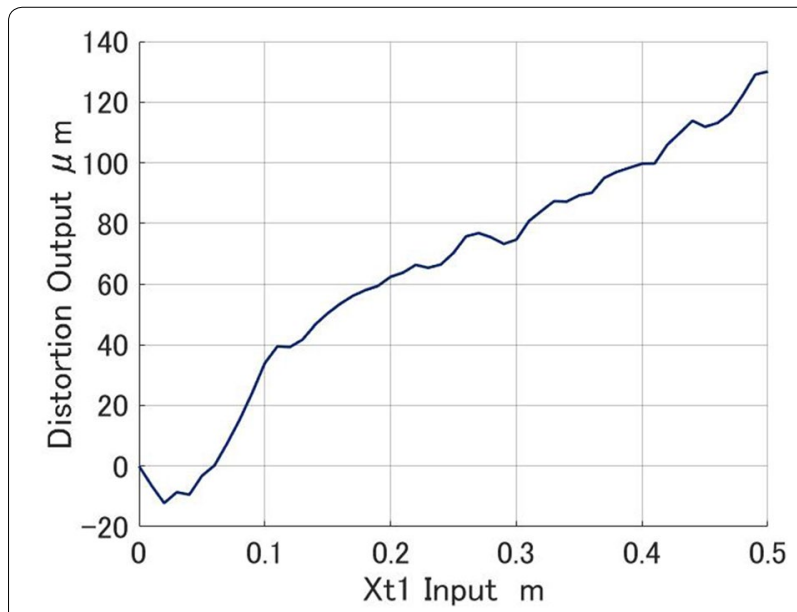

Fig. 3 Distortion of the gantry type linear motor slider

motor slider, $G_{1}(s), G_{2}(s), G_{3}(s)$ and $G_{4}(s)$ are transfer functions from each force reference to each acceleration, $s$ is Laplace operator, $x_{t 1}$ and $x_{t 2}$ are positions of two tables, "Approximation of distortion" block is same block used in Ref. [4] and [12] which outputs the measured distortion of Fig. 3, and $x_{f b 1}$ and $x_{f b 2}$ are positions of each table affected by the distortion.

\section{Identification of transfer functions [12, 13]}

By using the measured frequency responses, transfer functions which approximate the measured frequency responses were derived. Vibration of the experimental machine was excited by inputting a white noise signal to each linear motor and an acceleration signal was acquired from accelerometers which were attached to the top bar of the experimental machine [12]. Transfer functions $G_{i}$, which correspond to $G_{1}(s), G_{2}(s), G_{3}(s)$ and $G_{4}(s)$, are defined as described by Eq. (1).

$$
G_{i}(s)=\frac{b_{i 2} s^{2}+b_{i 1} s+b_{i 0}}{s^{2}+a_{i 1} s+a_{i 0}}
$$


In order to determine coefficients of $G_{i}(s)$, evaluation functions $J_{i}$ were defined as described by Eq. (2) for each $G_{i}(i=1,2,3$ and 4$)$, where $j$ is imaginary unit, $f_{k}[\mathrm{~Hz}]=0.5 \times k$ are frequency responses of $H^{i}$ with frequencies $f_{k}$, and $w_{i}\left(f_{k}\right)$ are weight functions, applied to each frequency.

$$
J_{i}=\sum_{k=0}^{400} w_{i}\left(f_{k}\right)\left|G_{i}\left(2 \pi j f_{k}\right)-H_{k}^{i}\right|^{2}
$$

Since $J_{i}$ represent the differences of frequency responses between $G_{i}$ and $H^{i}$, the optimal coefficients of $G_{i}$ were derived by minimizing $J_{i}$. In this case, values of the weight functions around $100 \mathrm{~Hz}$ were set larger than other weight values because all frequency responses have vibration mode at around $100 \mathrm{~Hz}$. Equation (3) shows the condition of the weight function $w_{i}\left(f_{k}\right)$.

$$
w_{i}\left(f_{k}\right)=\left\{\begin{array}{l}
100: 100 \leq f_{k} \leq 105 \\
10: 70 \leq f_{k}<100 \text { or } 105<f_{k} \leq 120 \\
1: 0<f_{k}<70 \text { or } 120<f_{k}
\end{array}\right.
$$

Table 2 shows the coefficients of transfer functions which are determined by this method. Figure 4 shows comparison of frequency responses $H^{i}$ and frequency responses of identified transfer functions $G_{i}$. Frequency responses of identified transfer functions $G_{i}$ does not

Table 2 Coefficients of identified transfer functions

\begin{tabular}{lllll}
\hline $\boldsymbol{i}$ & $\mathbf{1}$ & $\mathbf{2}$ & $\mathbf{3}$ & $\mathbf{4}$ \\
\hline$a_{i 1}$ & 151.83 & 117.84 & 115.93 & 138.88 \\
$a_{i 0}$ & $4.46 \times 10^{5}$ & $4.29 \times 10^{5}$ & $4.31 \times 10^{5}$ & $4.51 \times 10^{5}$ \\
$b_{i 2}$ & 3.77 & -0.159 & -0.157 & 3.79 \\
$b_{i 1}$ & 475.89 & 324.79 & 299.97 & 468.46 \\
$b_{i 0}$ & $7.95 \times 10^{5}$ & $6.25 \times 10^{5}$ & $6.24 \times 10^{5}$ & $7.76 \times 10^{5}$ \\
\hline
\end{tabular}
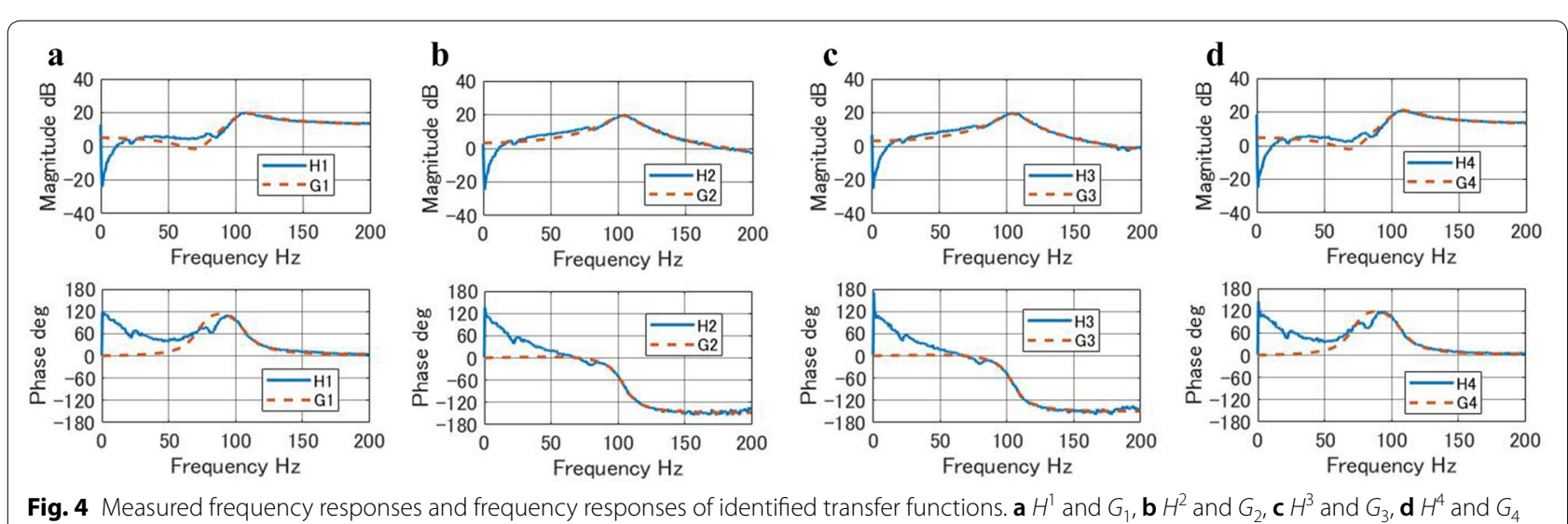

approximate the low-frequency region, but it can be confirmed that the vibration around $100 \mathrm{~Hz}$ existing in the experimental machine is well approximated. Therefore, this paper considers that each transfer function can approximate each frequency response.

\section{Modification of control model}

Since the two tables of the gantry-type linear motor slider are coupled by the top bar and the attachments, the position difference between the two tables does not exceed a certain range. Figure 5 shows the measured position difference when $40 \mathrm{~N}$ step force reference was input to one of the linear motor sliders [4]. A difference between (a) and (b) is a difference in input. According to these results, it is verified that position difference between the two tables without distortion converges to a certain value when step force input is applied to one of the linear motor sliders. However, the derived transfer function cannot reproduce this characteristic. Therefore, the transfer function needs to be modified.

In order to reproduce the characteristic caused by coupling of the two tables, the position difference between the two tables should converge to a certain value when
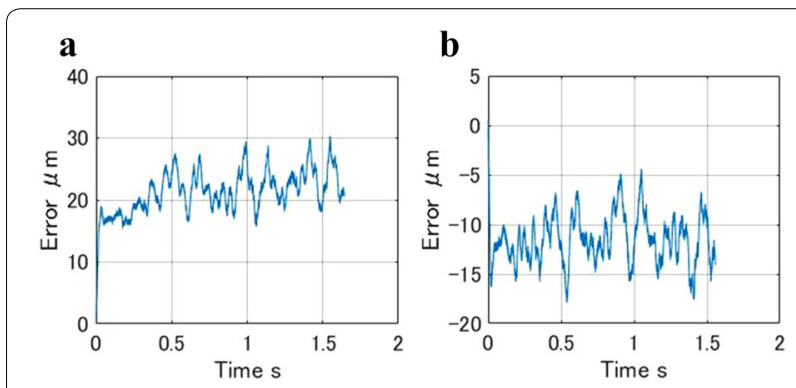

Fig. 5 Measured position difference between two tables. a Step force reference input to $F_{1}, \mathbf{b}$ Step force reference input to $F_{2}$ 
the input step force reference is applied to one of the two sliders in the control model.

According to the final value theorem, the final values of position difference for step force reference can be written as Eqs. (4) and (5). Two responses of the position differences to force reference $f_{1}(t)$ and $f_{2}(t)$ were defined as $e_{1}(t)$ and $e_{2}(t)$ respectively.

$$
\begin{aligned}
& \lim _{t \rightarrow \infty} e_{1}(t)=\lim _{s \rightarrow 0} s E_{1}(s)=\lim _{s \rightarrow 0} \frac{1}{s^{2}}\left(G_{1}-G_{2}\right) \\
& \lim _{t \rightarrow \infty} e_{2}(t)=\lim _{s \rightarrow 0} s E_{2}(s)=\lim _{s \rightarrow 0} \frac{1}{s^{2}}\left(G_{3}-G_{4}\right)
\end{aligned}
$$

To develop a control model which reproduces the characteristics caused by the tables coupling, Eqs. (4) and (5) should converge. Convergence conditions of these limits can be written as Eqs. (6)-(8) by using coefficients of transfer functions $G_{i}$ as shown in Eq. (1).

$$
\begin{aligned}
& a_{20} b_{10}-a_{10} b_{20}=0 \\
& a_{20} b_{11}+a_{21} b_{10}-a_{10} b_{21}-a_{11} b_{20}=0 \\
& a_{40} b_{30}-a_{30} b_{40}=0 \\
& a_{40} b_{31}+a_{41} b_{30}-a_{30} b_{41}-a_{31} b_{40}=0
\end{aligned}
$$

To satisfy these equations, coefficients of transfer functions in the control model were modified. In this case, not to change the frequency characteristics of the resonance point, poles of each transfer function should not be changed. Therefore, the coefficients of numerator of transfer function, $b_{11}, b_{20}, b_{30}$, and $b_{41}$ were replaced by $b_{11}^{\prime}, b^{\prime}{ }_{20}, b^{\prime}{ }_{30}$ and $b^{\prime}{ }_{41}$ as shown in Eqs. (10) to (13) respectively.

$$
\begin{aligned}
& b_{20}^{\prime}=\frac{a_{20} b_{10}}{a_{10}} \\
& b_{30}^{\prime}=\frac{a_{30} b_{40}}{a_{40}} \\
& b_{11}^{\prime}=\frac{1}{a_{20}}\left(a_{10} b_{21}+a_{11} b_{20}^{\prime}-a_{21} b_{10}\right) \\
& b_{41}^{\prime}=\frac{1}{a_{30}}\left(a_{40} b_{31}+a_{41} b_{30}^{\prime}-a_{31} b_{40}\right)
\end{aligned}
$$

Then the modified transfer functions were defined as $G^{\prime}{ }_{1}(s), G_{2}^{\prime}(s), G^{\prime}{ }_{3}(s)$ and $G^{\prime}{ }_{4}(s)$. Table 3 shows the coefficients of each modified transfer function. For the following discussion, the control model using $G^{\prime}{ }_{1}(s), G^{\prime}{ }_{2}(s)$,
Table 3 Coefficients of modified transfer functions

\begin{tabular}{lllll}
\hline $\boldsymbol{i}$ & $\mathbf{1}$ & $\mathbf{2}$ & $\mathbf{3}$ & $\mathbf{4}$ \\
\hline$a_{i 1}$ & 151.83 & 117.84 & 115.93 & 138.88 \\
$a_{i 0}$ & $4.46 \times 10^{5}$ & $4.29 \times 10^{5}$ & $4.31 \times 10^{5}$ & $4.51 \times 10^{5}$ \\
$b_{i 2}$ & 3.77 & -0.159 & -0.157 & 3.79 \\
$b_{i 1}$ & 389.89 & 324.79 & 299.97 & 344.46 \\
$b_{i 0}$ & $7.95 \times 10^{5}$ & $7.65 \times 10^{5}$ & $7.42 \times 10^{5}$ & $7.76 \times 10^{5}$ \\
\hline
\end{tabular}
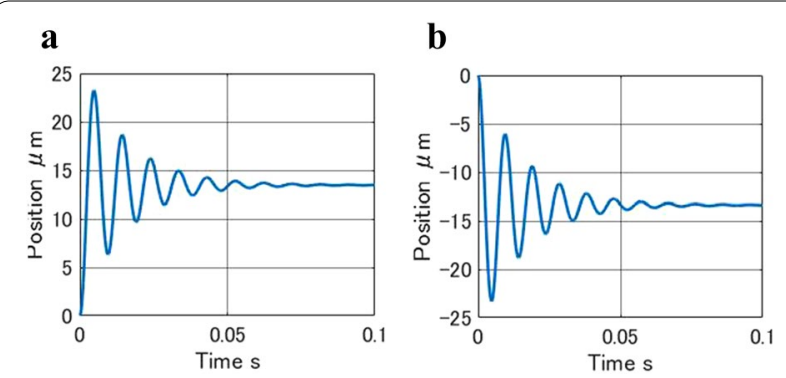

Fig. 6 Responses of $e_{1}^{\prime}(t)$ and $e_{2}^{\prime}(t)$ for step force reference. a Response of $e_{1}^{\prime}(t), \mathbf{b}$ Response of $e_{2}^{\prime}(t)$

$G^{\prime}{ }_{3}(s)$ and $G_{4}^{\prime}(s)$ instead of $G_{1}(s), G_{2}(s), G_{3}(s)$ and $G_{4}(s)$ is defined as a modified control model.

In the modified control model, responses of position difference for force references $f_{1}(t)$ and $f_{2}(t)$ were defined as $e_{1}^{\prime}(t)$ and $e_{2}^{\prime}(t)$ respectively.

Figure 6 shows the responses $e_{1}^{\prime}(t)$ and $e_{2}^{\prime}(t)$ for $40 \mathrm{~N}$ step force reference. It can be confirmed that both step responses converge to a certain value. Additionally, in comparison with results of actual experiment, shown in Fig. 5, convergence value is relatively close.

Figure 7 shows comparison between measured frequency response, frequency response of identified transfer function, and frequency response of modified transfer function. It can be seen that influence on frequency response by the coefficients modifications is small enough for all frequency responses and each $G^{\prime}{ }_{i}$ well approximates the measured frequency response.

Therefore, it can be said that modified control model which reproduces the characteristics of coupled tables was derived without changing frequency characteristics of the identified control model.

\section{Results and discussion}

\section{Verification of simulation model}

In order to verify the validity of the modified control model, comparison between the simulation results of modified control model and actual experimental results was conducted. In the previous research, the results of control experiments which used the same experimental 

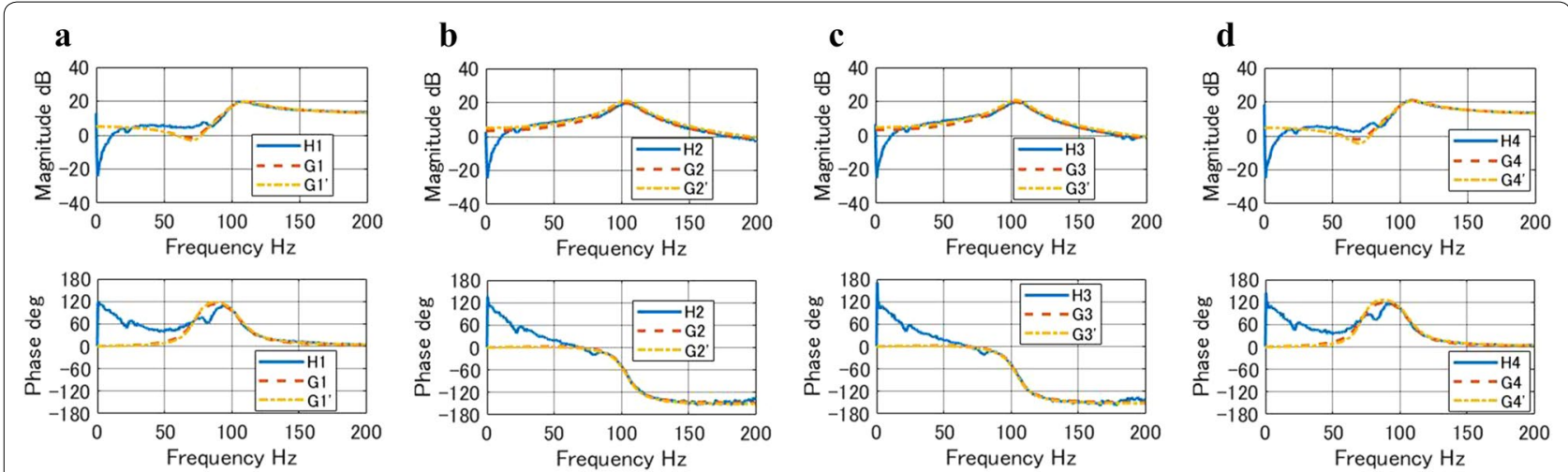

Fig. 7 Comparison of measured frequency responses $H^{\prime}$ and transfer functions $G_{i}$ and $G_{i}^{\prime} \mathbf{a} H^{1}, G_{1}$ and $G^{\prime}{ }_{1}, \mathbf{b} H^{2}, G_{2}$ and $G^{\prime}{ }_{2}, \mathbf{c} H^{3}, G_{3}$ and $G^{\prime}{ }_{3} \mathbf{d} H^{4}, G_{4}$ and $G_{4}^{\prime}$

Table 4 Parameters of friction

\begin{tabular}{lll}
\hline $\boldsymbol{n}$ & $\mathbf{1}$ & $\mathbf{2}$ \\
\hline$F_{s n}$ & $14.78 \mathrm{~N}$ & $16.43 \mathrm{~N}$ \\
$v_{s n}$ & $0.04 \mathrm{~m} / \mathrm{s}$ & $0.03 \mathrm{~m} / \mathrm{s}$ \\
$a_{n}$ & -6.67 & -7.42 \\
$F_{v n}$ & $34.09 \mathrm{~N} /(\mathrm{m} / \mathrm{s})$ & $36.68 \mathrm{~N} /(\mathrm{m} / \mathrm{s})$ \\
$F_{c n}$ & $9.92 \mathrm{~N}$ & $12.16 \mathrm{~N}$ \\
\hline
\end{tabular}

equipment as in this research were reported. In Ref. [4], P-PI control for each axis was applied for the control experiment and the results show that reaction force occurred between the two tables. In Ref. [14], a control method that suppresses reaction force was applied and the results show that the reaction force was suppressed with low feedback loop gain. However, with high feedback loop gain, vibration of force reference occurred.

In this research, control simulations were conducted with these two control methods and it was verified that the same phenomenon occurs in the simulations. The simulations were implemented by using MATLAB/Simulink programs.

Furthermore, the experimental machine of this study is the same as the experimental machine of Ref. [4], therefore, the same friction model is used as in Ref. [4]. Equation (14) shows the friction model and the Table 4 shows the parameters of friction.

$$
F_{d n}=\left\{\begin{array}{l}
F_{n}: \mathrm{if}\left|F_{n}\right| \leq F_{s n} \text { at } v_{t n}=0 \\
\operatorname{sgn}\left(v_{t n}\right) F_{s n} e^{-\left|v_{t n}\right| a_{n}: 0<\left|v_{t n}\right| \leq v_{s n}} \\
v_{t n} F_{v n}+\operatorname{sgn}\left(v_{t n}\right) F_{c n}: v_{s n}<\left|v_{t n}\right| \\
\quad(\mathrm{n}=1 \text { or } 2)
\end{array}\right.
$$

Here $F_{d n}$ is friction disturbance, $F_{s n}$ is maximum static friction force, $v_{t n}$ is table velocity, $a$ is power index to

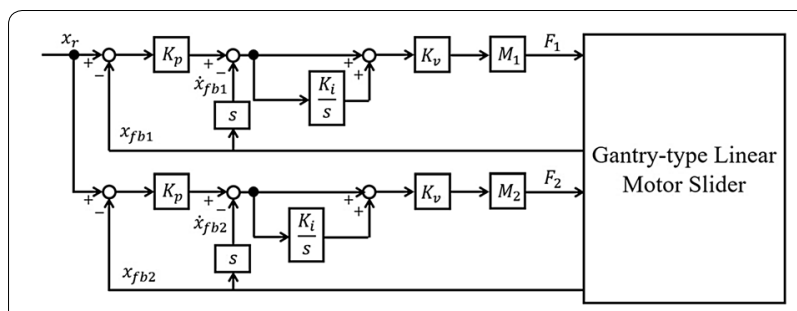

Fig. 8 Block diagram of Control method 1

express friction characteristic in a region below Stribeck velocity, $v_{s n}$ is Stribeck velocity, $F_{\mathrm{vn}}$ is viscous friction coefficient, and $F_{c n}$ is Coulomb friction.

\section{Simulation with control method 1 [4]}

As the control method 1, proportional-position-proportional-integral-velocity (P-PI) control for each axis, as shown in Ref. [4] was used. Therefore, the force references for each axis are shown in Eqs. (15) and (16). Figure 8 shows block diagram of control method 1 .

$$
\begin{aligned}
F_{1}= & M_{1}\left[K_{v}\left\{K_{p}\left(x_{r}-x_{f b 1}\right)-\dot{x}_{f b 1}\right\}\right. \\
& \left.+K_{i} \int K_{v}\left\{K_{p}\left(x_{r}-x_{f b 1}\right)-\dot{x}_{f b 1}\right\} d t\right] \\
F_{2}= & M_{2}\left[K_{v}\left\{K_{p}\left(x_{r}-x_{f b 2}\right)-\dot{x}_{f b 2}\right\}\right. \\
& \left.+K_{i} \int K_{v}\left\{K_{p}\left(x_{r}-x_{f b 2}\right)-\dot{x}_{f b 2}\right\} d t\right]
\end{aligned}
$$

where $M_{1}$ and $M_{2}$ are nominal masses of each table, $x_{f b 1}$ and $x_{f b 2}$ are position responses of each table, $K_{p}$ is position proportional gain, $K_{i}$ is velocity integral gain, $K_{v}$ is 
Table 5 Feedback loop gain for control method 1

\begin{tabular}{llll}
\hline & $\boldsymbol{K}_{\boldsymbol{p}}$ & $\boldsymbol{K}_{\boldsymbol{v}}$ & $\boldsymbol{K}_{\boldsymbol{i}}$ \\
\hline Case 1-1 & $251 / \mathrm{s}$ & $1001 / \mathrm{s}$ & $1001 / \mathrm{s}$ \\
Case 1-2 & $501 / \mathrm{s}$ & $2001 / \mathrm{s}$ & $2001 / \mathrm{s}$ \\
\hline
\end{tabular}

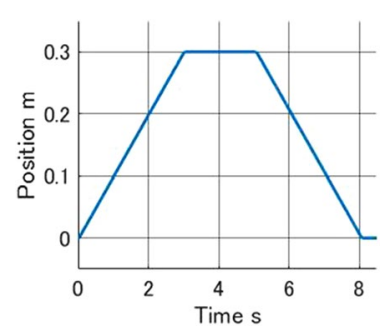

Fig. 9 Position reference

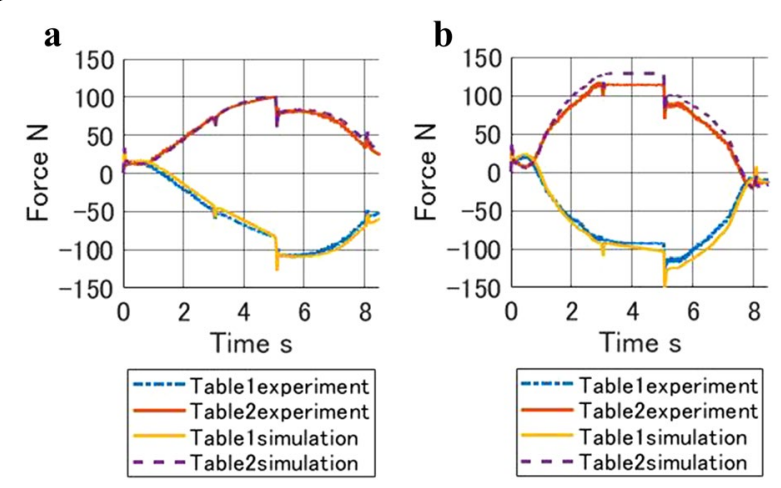

Fig. 10 Results of simulation and comparison with experimental results in control method 1. a Case 1-1, b Case1-2

velocity proportional gain, and $x_{r}$ is position reference, $s$ is Laplacian operator.

In this simulation, position reference and feedback loop gain are set to the same values as in Ref. [4]. The conditions of feedback loop gain are shown in Table 5 and position reference is shown in Fig. 9.

Figure 10 shows results of the simulation and comparison with the experimental results. Figure 10 shows that reaction forces between Tables 1 and 2 occurred also in the simulation. Simulation result has the same characteristics of experimental result. Therefore, under the conditions of control method 1, the control model approximates the actual experimental results.

\section{Simulation with control method $2[10,14]$}

As the control method 2, a method that suppresses the reaction force, which is shown in Ref. [14] was used. Therefore, force references for each linear motor are shown in Eqs. (17) and (18), where $l$ is distance between

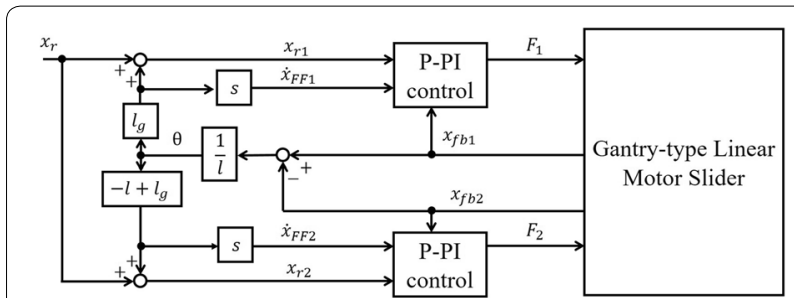

Fig. 11 Block diagram of Control method 2

Table 6 Feedback loop gain for control method 2

\begin{tabular}{llll}
\hline & $\boldsymbol{K}_{\boldsymbol{p}}$ & $\boldsymbol{K}_{\boldsymbol{v}}$ & $\boldsymbol{K}_{\boldsymbol{i}}$ \\
\hline Case 2-1 & $1001 / \mathrm{s}$ & $4001 / \mathrm{s}$ & $4001 / \mathrm{s}$ \\
Case 2-2 & $125 \mathrm{1} / \mathrm{s}$ & $5001 / \mathrm{s}$ & $5001 / \mathrm{s}$ \\
\hline
\end{tabular}

Tables 1 and $2, l_{g}$ is distance between Table 1 and center of the mass, and $\theta$ is rotation angle. Other parameters have the same meaning as in control method 1. Control method 2 is based on the P-PI control. Hence, it considers $\theta$ but has no control over it, paying closer attention to the work-point position on the top bar of the linear motor slider, which in general is the center-of-mass of the top bar. Figure 11 shows block diagram of control method 2 .

$$
\begin{aligned}
F_{1}= & M_{1}\left[K_{v}\left\{K_{p}\left(x_{r 1}-x_{f b 1}\right)-\dot{x}_{f b 1}+\dot{x}_{F F 1}\right\}\right. \\
& \left.+K_{i} \int K_{v}\left\{K_{p}\left(x_{r 1}-x_{f b 1}\right)-\dot{x}_{f b 1}+\dot{x}_{F F 1}\right\} d t\right] \\
F_{2}= & M_{2}\left[K_{v}\left\{K_{p}\left(x_{r 2}-x_{f b 2}\right)-\dot{x}_{f b 2}+\dot{x}_{F F 2}\right\}\right. \\
& \left.+K_{i} \int K_{v}\left\{K_{p}\left(x_{r 2}-x_{f b 2}\right)-\dot{x}_{f b 2}+\dot{x}_{F F 2}\right\} d t\right]
\end{aligned}
$$

where

$$
\begin{aligned}
& x_{r 1}=x_{r}+l_{g} \theta \\
& x_{r 2}=x_{r}-\left(l-l_{g}\right) \theta \\
& \dot{x}_{F F 1}=l_{g} \dot{\theta} \\
& \dot{x}_{F F 2}=-\left(l-l_{g}\right) \dot{\theta} \\
& \theta=\frac{x_{f b 1}-x_{f b 2}}{l}
\end{aligned}
$$



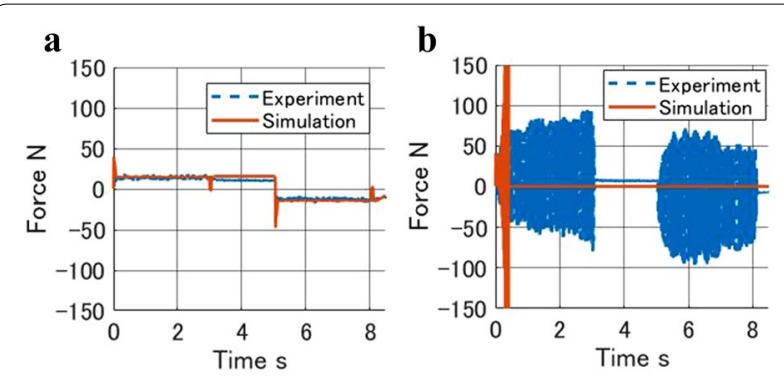

Fig. 12 Results of simulation and comparison with experimental results in control method 2. a Case2-1, b Case 2-2

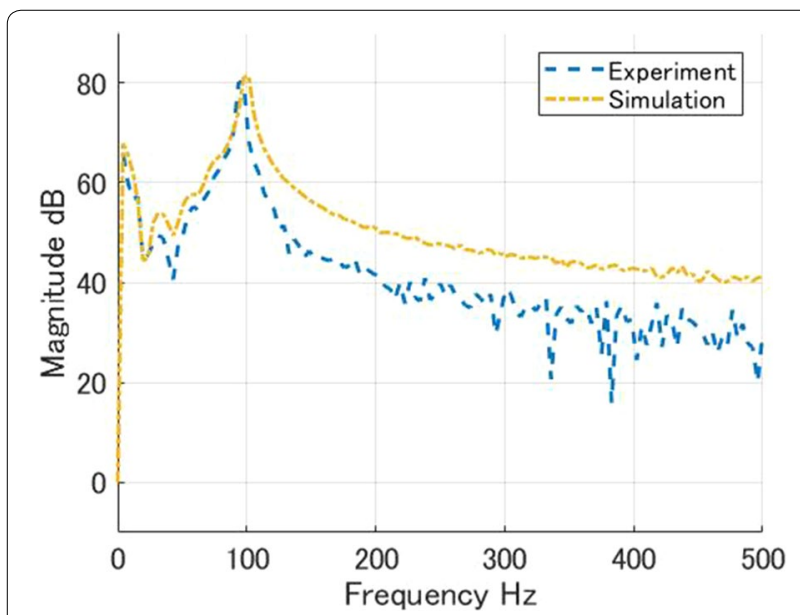

Fig. 13 Amplitude spectrum of force reference $F_{1}$ in Case 2-2

The conditions of feedback loop gain are shown in Table 6 and position reference is shown in Fig. 9. In this simulation, if the force reference exceeds $500 \mathrm{~N}$, the simulation stops automatically. Figure 12 shows the results of the simulation and comparison with the experimental results. In Fig. 12, only $F_{1}$ is shown as a force reference because $F_{1}$ and $F_{2}$ are exactly the same. In case 2-1, in both simulation and experiment, the reaction forces between the two tables were suppressed. Moreover, the force reference is substantially the same as the experimental result. In case 2-2, the force reference vibrates. In the simulation, the force reference diverged rapidly, and the simulation stopped because magnitude of the force reference exceeded $500 \mathrm{~N}$. However, in the actual experiment, the force reference vibrated within the range of $\pm 100 \mathrm{~N}$. To analyze the characteristics of force reference vibration, Fast-Fourier-Transformation was applied, and amplitude spectrum was acquired. Figure 13 shows amplitude spectrum of force reference $F_{1}$ in case 2-2. According to the amplitude spectrum, it can be seen that each force reference vibrates at the same frequency of $100 \mathrm{~Hz}$. Additionally, based on the position response, a velocity response of actual experiment was calculated by using Euler approximation. Figure 14 shows velocity response in the simulation and in the actual experiment. Figure 14b, d can be seen that vibrations of $V_{f b 1}$ and $V_{f b 2}$ are in phase. Therefore, the signal values in Figs. 12 and 14 are different between the experimental results and the simulation results. However, experimental results and simulation results have the same tendency. In Case 1, the slider operates safely, but in Case 2, vibration occurs. In every condition of feedback loop gain, all simulation results in control method 2 are similar to the experimental results.

\section{Conclusion}

In this research, highly precise control model that can reproduce all frequency characteristics and unique characteristics of a large-sized gantry-type linear motor slider was derived. According to comparison of simulations and actual experiments which were implemented with two control methods, the control model well approximates characteristics of a gantry-type linear motor slider. A control method for improving the control performance of large-sized gantry-type linear motor slider can be derived by using this model. Future study topic is verification of
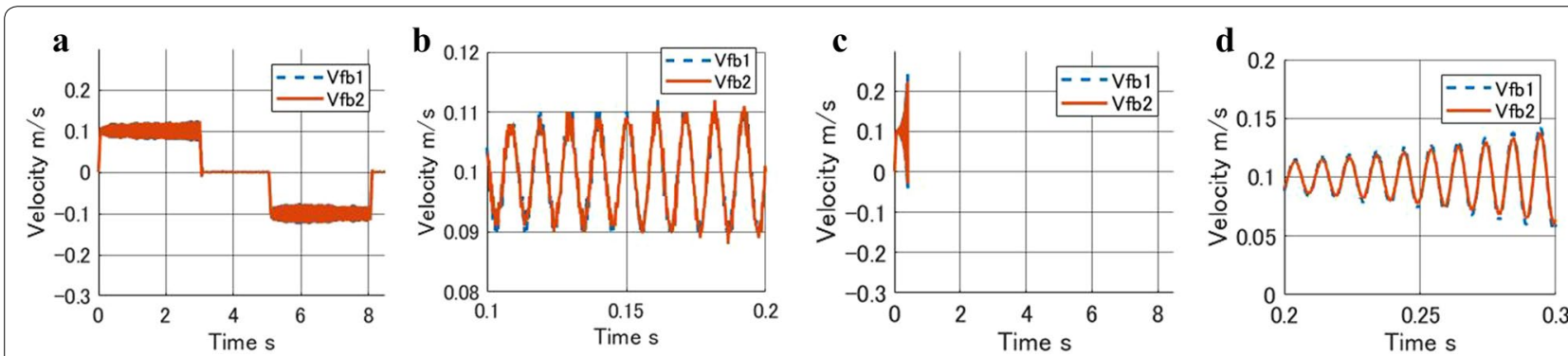

Fig. 14 Comparison of velocity response of simulation and actual experiment in Case 2-2. a Velocity response of actual machine, $\mathbf{b}$ velocity response of actual machine (enlarged), c velocity response of simulation, $\mathbf{d}$ velocity response of simulation (enlarged) 
validity of the proposed method when characteristics of actual machine change, for example, the movement of the center of gravity in real time.

\section{Acknowledgements}

Not applicable.

\section{Authors' contributions}

TO and TT developed the system and carried out the experiments and simulations. $\mathrm{HHo}$, SW and KT managed the study. HHa managed the experiments. All members verified the content of their contributions. All authors read and approved the final manuscript.

\section{Funding}

Not applicable.

\section{Availability of data and materials}

The datasets used and/or analyzed in this study are available from the corresponding author on request.

\section{Competing interests}

The authors declare that they have no competing interests.

\section{Author details}

${ }^{1}$ Department of Mechanical Engineering, Faculty of Science and Engineering, Kyushu Sangyo University, 2-3-1, Matsukadai, Higashi-ku, Fukuoka 813-8503, Japan. ${ }^{2}$ Research Institute of Systems Planning, Inc., 18-6 Japan Hall, Sakuragaokacho, Shibuya-ku, Tokyo 150-0031, Japan. ${ }^{3}$ Department of Biological Functions Engineering, Graduate School of Life Science and Systems Engineering, Kyushu Institute of Technology, 2-4, Hibikino, Wakamatsu-ku, Kitakyushu 808-0196, Japan. ${ }^{4}$ Department of Mechanical Engineering, National Institute of Technology, Kagoshima College, 1460-1 Shinko, Hayato-cho, Kirishima 899-5193, Japan. ${ }^{5}$ Department of Creative Engineering, National Institute of Technology, Kitakyushu College, 5-20-1 Shii, Kokuraminami-ku, Kitakyushu 802-0985, Japan.

Received: 19 December 2019 Accepted: 23 May 2020

Published online: 28 May 2020

\section{References}

1. Haidong Y, Babak F (2010) Industrial servo applications of linear induction motors based on dynamic maximum force control. In: 2010 twenty-fifth annual IEEE applied power electronics conference and exposition (APEC), Palm Springs, CA, 21-25 Feb; 2010
2. Nakamura Y, Morimoto K, Wakui S (2011) Position control of linear slider via feedback error learning. In: 2011 third pacific-asia conference on circuits, communications and system (PACCS), Wuhan, China, 17-18 July 2011

3. Yamada T, Fujisaki K (2009) Application of linear induction motor for tension supply and heating to thin sheet steel. Electr Eng J.jn 168(2):38-47

4. Ojiro T, Honda H, Tsuruta K, Hanamoto T (2018) Control model for largesized gantry type linear motor slider. Electr Eng Jpn 203(2):39-46

5. Nakashima R, Hao S, Honda H, Oguro R, Miyakawa H, Tsuji T (2003) Position control for a linear slider with twin linear drives. Electr Eng J.pn 147(4):68-76

6. Sato K, Tsuruta K, Kikuchi T, Honda H (2008) A robust adaptive control for parallel linear sliders using decoupling model. In: 2008 SICE annual conference, Tokyo, Japan, 20-22 Aug; 2008

7. Terasaki F, Kobayashi J, Oguro R, Ohkawa F (2006) A positioning control of a serial twin linear slider system with machine stand vibration. In: 2006 IEEE international conference on industrial technology, Mumbai, India 15-17 Dec. 2006

8. Tomita Y, Kojima E, Kawachi S, Koyanagawa Y, Ootsuka S (2012) Development and applications of sumitomo precision stage technologies for FPD process. J Jpn Soc Precis Eng 78(2):117-121

9. Sogabe M, Iwashita Y, Sonoda N, Kakino Y (2007) A study on the servostability of the tandem driven machine with linear motors. J Jpn Soc Precis Eng 73(5):605-610

10. Ojiro T, Tachibana T, Honda H, Hamamatsu H, Tsuruta K, Hanamoto T (2019) Consideration of multi-degree of freedom vibration on large-sized gantry type linear motor slider. J Robot Mechatr JRM 31(2):240-250

11. Watanabe S, Oguro R, Kobayashi J, Ohkawa F (2006) A decoupling method for serial twin linear slider system with machine stand vibration. Proc IEEE Int Symp Ind Electron 2006:3032-3037

12. Tachibana T, Ojiro T, Hamamatsu H, and Honda H(2019) Study on Control Model Structuring of Large-Sized Gantry-Type Linear Motor Slider by Measuring Frequency Response. The 5th IEEJ international workshop on Sensing, Actuation, Motion Control, and Optimization (SAMCON2019) TT2-2

13. Levy EC (1959) Complex-curve fitting. In: IRE transaction on automatic control. AC-4. pp 37-44

14. Ojiro T, Honda H, Hanamoto T, Tsuruta K (2017) Consideration on control method of the large-sized gantry-type linear motor slider. In: 2017 IEEE international conference on mechatronics and automation (ICMA). pp $1198-1203$

\section{Publisher's Note}

Springer Nature remains neutral with regard to jurisdictional claims in published maps and institutional affiliations.

\section{Submit your manuscript to a SpringerOpen ${ }^{\circ}$ journal and benefit from:}

- Convenient online submission

- Rigorous peer review

- Open access: articles freely available online

- High visibility within the field

- Retaining the copyright to your article

Submit your next manuscript at $\boldsymbol{\nabla}$ springeropen.com 